
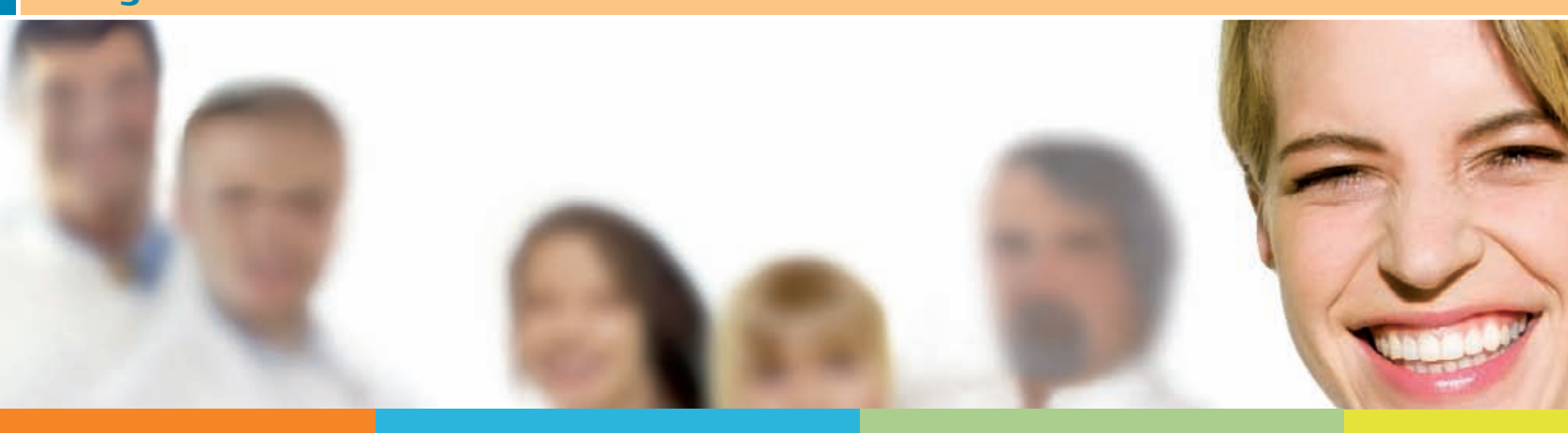

\title{
Der Verband für alle Berufsgruppen aus dem Arbeitsfeld Nephrologie Die Arbeitsgemeinschaft \\ für nephrologisches Personal e. V.
}

Die AfnP ist mit über 1500 Mitgliedern der größte nationale Berufsverband für die nephrologische Pflege. Selbstverständlich finden auch andere Berufsgruppen aus dem Tätigkeitsfeld der Nephrologie und Nierenersatztherapie sowie verwandte Verfahren in der AfnP einen Ansprechpartner. In der 30-jährigen Verbandsgeschichte hat die AfnP wichtige Ziele erreicht und viele Anliegen ihrer Mitglieder realisiert.

Wir nehmen maßgeblichen Einfluss auf Veränderungen und Entwicklungen in der Nephrologie und Dialyse. Dies ist nur in Kooperation mit den ärztlichen Fachgesellschaften und gesellschaftlich relevanten Institutionen möglich. Ständiger Dialog und die Kooperation mit anderen Verbänden kennzeichnen unsere Arbeitsweise.

Wir transportieren die Ideen unserer Mitglieder an die richtigen Adressen Stellvertretend für das Erreichte steht die Fachweiterbildung Fachkrankenpflege Nephrologie, an deren Implementierung die AfnP maßgeblich beteiligt war. Als Mitglied in der Bundesarbeitsgemeinschaft der nephrologischen Weiterbildungsstätten und in

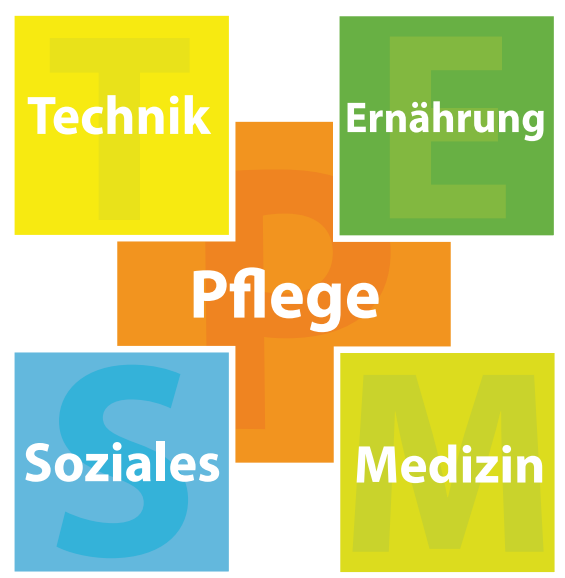

der Arbeitsgruppe der DKG (Deutsche Krankenhausgesellschaft) wird diese Arbeit energisch fortgesetzt.

Nur gemeinsam werden wir gehört Gemeinsam mit dem Arbeitskreis für angewandte Hygiene erarbeiten wir die Inhalte der „Leitlinie für angewandte Hygiene in Dialyseeinheiten“. Abgesehen davon engagieren wir uns in zahlreichen weiteren Projekten.

Jährlich findet im Oktober das AfnP-Symposium in Fulda statt. Darüber hinaus bieten wir interessante Fortbildungsveranstaltungen, die von unseren Ländervertretern organisiert und betreut werden. Die Ländervertreter der einzelnen Bundesländer stehen Ihnen für Fragen, Anregungen und Informationen (z. B. über regionale Fortbildungsangebote) jederzeit gerne zur Verfügung. Die Kontaktadressen und weitere Informationen erhalten Sie in unserer Geschäftsstelle oder auf unserer Internetpräsenz unter www.afnp.de.

Ihre AfnP

\section{Unsere Ziele}

- Förderung der Fort- und Weiterbildung

- Verbesserung der Struktur-, Prozessund Ergebnisqualität in der Versorgung und Betreuung von nierenkranken Menschen

- Erweiterung des Informationsaustausches zwischen allen beteiligten und kooperierenden Partnern

- konstruktive Kommunikation mit privaten, kommunalen und staatlichen Stellen

\section{Eine Mitgliedschaft, die sich lohnt!}

Der Mitgliedsbeitrag beträgt nur 25 Euro pro Kalenderjahr. Dieser Betrag gilt satzungsgemäß für alle Berufsgruppen, die in der Nephrologie und Dialyse tätig sind. Wir bieten unseren Mitgliedern:

- kostenlose Teilnahme am jährlichen Symposium (Nichtmitglieder 60 Euro)

- 10-mal jährlich kostenloser Bezug der Zeitschrift Dialyse aktuell ab 2009

- eine CD mit allen Beiträgen des Symposiums

- preiswerte Seminare mit kompetenten Referenten und interessanten Themen (Nichtmitglieder zahlen einen deutlich höheren Teilnehmerbeitrag)

Wollen Sie Mitglied werden? Einen Mitgliedsantrag finden Sie auf unserer Homepage oder Sie wenden sich schriftlich an unsere Geschäftsstelle.

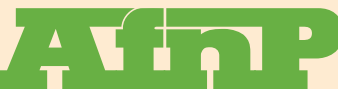

Arbeitsgemeinschaft für nephrologisches Personal e.V.
Geschäftsstelle der AfnP e. V.

Käppelesweg 8, 89129 Langenau

Tel.: 07345/22933, Fax: 07345/7540

E-Mail: info@afnp.de, Homepage: www.afnp.de 